\title{
ALGEBRAIC PROPERTIES OF BI-POLYMATROIDAL IDEALS
}

\author{
M. LA BARBIERA
}

\begin{abstract}
Classes of monomial ideals are considered in the polynomial ring in two sets of variables $R=K\left[X_{1}, \ldots, X_{n} ; Y_{1}, \ldots, Y_{m}\right]$. Some algebraic properties of bipolymatroidal ideals of $R$ are studied. More precisely, the behavior of the monomial localization of such ideals is investigated.
\end{abstract}

\section{INTRODUCTION}

Let $R=K\left[X_{1}, \ldots, X_{n} ; Y_{1}, \ldots, Y_{m}\right]$ be the polynomial ring in two sets of variables over a field $K$. Classes of monomial ideals of $R$ have been studied intensively (see [10]). In this paper, we are interested in a special class of monomial ideals of $R$, namely, in the bi-polymatroidal ideals. In [7, the author introduced the generalized notion of a discrete bi-polymatroid and the corresponding notion of a bi-polymatroidal ideal in $R$. Let $L$ be a monomial ideal of $R$ generated in a single degree, and let $G(L)$ be its unique set of minimal generators. $L$ is called a bi-polymatroidal ideal if for any two monomials $u, v \in G(L)$ such that $\operatorname{deg}_{X_{i}}(u)>\operatorname{deg}_{X_{i}}(v)$ or $\operatorname{deg}_{Y_{k}}(u)>\operatorname{deg}_{Y_{k}}(v)$, there exists $j \in[n]$ with $\operatorname{deg}_{X_{j}}(u)<\operatorname{deg}_{X_{j}}(v)$ or $l \in[m]$ with $\operatorname{deg}_{Y_{l}}(u)<\operatorname{deg}_{Y_{l}}(v)$ such that $X_{j}\left(u / X_{i}\right) \in G(L)$ or $Y_{l}\left(u / Y_{k}\right) \in G(L)$. An interesting class of discrete bi-polymatroids is the discrete bi-polymatroid of Veronese type, whose set of bases is

$$
B_{q, s}=\left\{(a ; b) \in \mathbb{Z}_{+}^{n+m}:|a|=k,|b|=r, 0 \leq a_{i}, b_{j} \leq s\right\}, \quad s \leq q .
$$

The corresponding bi-polymatroidal ideals of $R$ are the ideals of Veronese bi-type $L_{q, s}$. They are an extension of the ideals of Veronese type in a polynomial ring in two sets of variables. More precisely, the Veronese bi-type ideals are monomial ideals of $R$ generated in the same degree: $L_{q, s}=\sum_{k+r=q} I_{k, s} J_{r, s}$, with $k, r \geq 1, s \leq q$, where $I_{k, s}$ is the Veronese-type ideal generated in degree $k$ by the set

$$
\left\{X_{1}^{a_{i_{1}}} \ldots X_{n}^{a_{i_{n}}} \mid \sum_{j=1}^{n} a_{i_{j}}=k, 0 \leq a_{i_{j}} \leq s, s \in\{1, \ldots, k\}\right\}
$$

and $J_{r, s}$ is the Veronese-type ideal generated in degree $r$ by the set

$$
\left\{Y_{1}^{b_{i_{1}}} \ldots Y_{m}^{b_{i_{m}}} \mid \sum_{j=1}^{m} b_{i_{j}}=r, 0 \leq b_{i_{j}} \leq s, s \in\{1, \ldots, r\}\right\},
$$

see [5, 6, 7, 8].

Now we are interested in a problem introduced in [1] and [4, where the authors proved that a monomial localization of a polymatroidal ideal is a polymatroidal ideal.

Let $\wp$ be a prime ideal of $R$. The monomial localization of a monomial ideal $L$ with respect to $\wp$ is the monomial ideal $L(\wp)$ obtained from $L$ by substituting 1 for the variables that do not belong to $\wp$. More precisely, setting $\wp=\left(X_{i_{1}}, \ldots, X_{i_{r}}, Y_{j_{1}}, \ldots, Y_{j_{t}}\right)$, we

2010 Mathematics Subject Classification. Primary 13F20.

Key words and phrases. Bi-polymatroidal ideals, monomial localization. 
denote by $L(\wp)$ the monomial ideal in the polynomial $\operatorname{ring} K\left[X_{i_{1}}, \ldots, X_{i_{r}}, Y_{j_{1}}, \ldots, Y_{j_{t}}\right]$, where $L(\wp)=L_{C}$ with $C=[n+m] \backslash\left\{i_{1}, \ldots, i_{r}, j_{1}, \ldots, j_{t}\right\}$. If $L$ is a square-free monomial ideal, then $L(\wp)=L: X_{C}$, where $X_{C}=\prod_{i \in C} X_{i}$.

In this paper, we generalize the properties studied in 1 about the monomial localization to all bi-polymatroidal ideals of $R$; in particular, we verify that a monomial localization $L(\wp)$ of a bi-polymatroidal ideal $L$ is again a bi-polymatroidal ideal.

The paper is organized as follows. In $\S 1$, some preliminary notions on the bi-polymatroidal ideals are given. In $\S 2$ we study a certain permanence property of the bi-polymatroidal ideals of $R$. We show that the property of $L$ to be a bi-polymatroidal ideal is equivalent to the fact that $L: u$ is a bi-polymatroidal ideal for all monomials $u$ of $R$. Moreover, we prove that a monomial localization of a bi-polymatroidal ideal is again a bi-polymatroidal ideal.

\section{§1. Preliminary notions}

Let $n, m>0$ be integers, and let $[n+m]=\{1,2, \ldots, n+m\}$. Let $\mathbb{Z}_{+}$be the set of nonnegative integers and $\mathbb{Z}_{+}^{n+m}$ the set of the vectors $(a ; b)$ with $a \in \mathbb{Z}_{+}^{n}$ and $b \in \mathbb{Z}_{+}^{m}$, i.e., $(a ; b)=\left(a_{1}, \ldots, a_{n} ; b_{1}, \ldots, b_{m}\right) \in \mathbb{Z}_{+}^{n+m}$ with $a_{i} \geq 0, b_{j} \geq 0$ for all $i, j$.

The modulus of the vector $(a ; b)$ is the number $|(a ; b)|=|a|+|b|=\sum_{i=1}^{n} a_{i}+\sum_{j=1}^{m} b_{j}$. Let $(a ; b)$ and $(c ; d)$ be two vectors of $\mathbb{Z}_{+}^{n+m}$, we have $(a ; b) \geq(c ; d)$ if all components $\left(a_{i}-c_{i} ; b_{i}-d_{i}\right)$ of the vector $(a-c ; b-d)$ are nonnegative. If $(a ; b) \geq(c ; d)$ and $(a ; b) \neq(c ; d)$, we write $(a ; b)>(c ; d)$. We say that $(c ; d)$ is a subvector of $(a ; b)$ if $(a ; b) \geq(c ; d)$. Moreover, we set $(a ; b) \vee(c ; d)=\left(\max \left\{a_{1}, c_{1}\right\}, \ldots, \max \left\{a_{n}, c_{n}\right\}\right.$; $\left.\max \left\{b_{1}, d_{1}\right\}, \ldots, \max \left\{b_{m}, d_{m}\right\}\right)$.

Hence $(a ; b) \leq(a ; b) \vee(c ; d)$ and $(c ; d) \leq(a ; b) \vee(c ; d)$.

In [3], the following combinatorial concept of a discrete polymatroid was introduced.

Definition 1.1. A discrete polymatroid on the set $[n]=\{1,2, \ldots, n\}$ is a nonempty finite subset $P \subset \mathbb{Z}_{+}^{n}$ satisfying the following conditions:

1) together with each $u \in P$, the set $P$ contains all its integral subvectors, that is, if $u \in P$ and $v \in \mathbb{Z}_{+}^{n}$ with $v \leq u$, then $v \in P$;

2) for all $u, v \in P$ with $|u|<|v|$, there is a vector $w \in P$ such that $u<w<u \vee v$.

In [7, the following notion of a discrete bi-polymatroid was introduced as a generalization of the discrete polymatroid.

Definition 1.2. A discrete bi-polymatroid on the set $[n+m]$ is a nonempty finite subset $P \subset \mathbb{Z}_{+}^{n+m}$ satisfying the following conditions:

$1)$ together with each $(a ; b) \in P$, the set $P$ contains all its integral subvectors, that is, if $(a ; b) \in P$ and $(c ; d) \in \mathbb{Z}_{+}^{n+m}$ with $(c ; d) \leq(a ; b)$, then $(c ; d) \in P$;

2) for all $(a ; b),(c ; d) \in P$ with $|(a ; b)|<|(c ; d)|$, there is a vector $(u ; v) \in P$ such that $(a ; b)<(u ; v)<(a ; b) \vee(c ; d)$.

A base of a discrete bi-polymatroid $P$ is a vector $(a ; b) \in P$ such that $(a ; b)<(c ; d)$ for no $(c ; d) \in P$. The set of bases of $P$ is denoted by $B(P)$.

Remark 1.3. Each base of a discrete bi-polymatroid $P$ has the same modulus, called the rank of $P$. Indeed, if $(a ; b)$ and $(c ; d)$ are bases of $P$ with $|(a ; b)|<|(c ; d)|$, then by Definition 1.2 there exists $(u ; v) \in P$ with $(a ; b)<(u ; v)<(a ; b) \vee(c ; d)$. This contradicts the maximality of $(a ; b) \in B(P)$.

A characterization of discrete bi-polymatroids in terms of their set of bases looks like this. 
Theorem 1.4 (Bi-exchange property, see [7]). $P$ is a discrete bi-polymatroid if and only if the following is true: if $(a ; b),(c ; d) \in B(P)$ with $a_{i}>c_{i}$ or $b_{k}>d_{k}$, then there exist $j \in\{1, \ldots, n\}$ and $l \in\{1, \ldots, m\}$ with $a_{j}<c_{j}$ or $b_{l}<d_{l}$ such that $(a ; b)-\left(e_{i} ; 0\right)+\left(e_{j} ; 0\right) \in$ $B(P)$ or $(a ; b)-\left(0 ; e_{k}^{\prime}\right)+\left(0 ; e_{l}^{\prime}\right) \in B(P)$, where the $e_{i}, e_{j}^{\prime}$ denote the standard basis vectors of $\mathbb{Z}_{+}^{n}, \mathbb{Z}_{+}^{m}$, respectively.

Let $R=K\left[X_{1}, \ldots, X_{n} ; Y_{1}, \ldots, Y_{m}\right]$ be the polynomial ring in two sets of variables with $\operatorname{deg}\left(X_{i}\right)=\operatorname{deg}\left(Y_{j}\right)=1$ for all $i=1, \ldots, n, j=1, \ldots, m$. For a monomial ideal $L \subset R$, we denote by $G(L)$ its unique set of minimal generators.

A monomial ideal generated by all the monomials corresponding to the set $B(P)$ of bases of a discrete bi-polymatroid is called a bi-polymatroidal ideal and is generated by all the monomials $\underline{X}^{a} \underline{Y}^{b}$ with $(a, b) \in B(P)$, where $\underline{X}^{a} \underline{Y}^{b}$ stands for $X_{1}^{a_{1}} \ldots X_{n}^{a_{n}} Y_{1}^{b_{1}} \ldots Y_{m}^{b_{m}}$.

In [7, the following definition of a bi-polymatroidal ideal was given as a consequence of Theorem 1.4.

Definition 1.5. A monomial ideal $L$ of $R$ generated in a single degree is said to be $b i$ polymatroidal if the following condition is satisfied: for all monomials $u=X_{1}^{a_{1}} \ldots X_{n}^{a_{n}} Y_{1}^{b_{1}}$ $\ldots Y_{m}^{b_{m}}$ and $v=X_{1}^{c_{1}} \ldots X_{n}^{c_{n}} Y_{1}^{d_{1}} \ldots Y_{m}^{d_{m}}$ in $G(L)$ and for each $i$ with $a_{i}>c_{i}$ or $k$ with $b_{k}>d_{k}$, we have $j \in[n]$ with $a_{j}<c_{j}$ or $l \in[m]$ with $b_{l}<d_{l}$ such that $X_{j}\left(u / X_{i}\right) \in G(L)$ or $Y_{l}\left(u / Y_{k}\right) \in G(L)$.

It follows that for any two monomials $u, v \in G(L) \operatorname{such}$ that $\operatorname{deg}_{X_{i}}(u)>\operatorname{deg}_{X_{i}}(v)$ or $\operatorname{deg}_{Y_{k}}(u)>\operatorname{deg}_{Y_{k}}(v)$ there exist $j \in[n]$ with $\operatorname{deg}_{X_{j}}(u)<\operatorname{deg}_{X_{j}}(v)$ or $l \in[m]$ with $\operatorname{deg}_{Y_{l}}(u)<\operatorname{deg}_{Y_{l}}(v)$ such that $X_{j}\left(u / X_{i}\right) \in G(L)$ or $Y_{l}\left(u / Y_{k}\right) \in G(L)$.

Theorem 1.6 (see [7]). The bi-polymatroidal ideals of $R=K\left[X_{1}, \ldots, X_{n} ; Y_{1}, \ldots, Y_{m}\right]$ have linear quotients.

We shall describe a special class of bi-polymatroidal ideals. Let $q, s, r, k$ be nonnegative integers such that $k+r=q$ and $s \leq q$; then

$$
B_{q, s}=\left\{(a ; b) \in \mathbb{Z}_{+}^{n+m}:|a|=k,|b|=r, 0 \leq a_{i}, b_{j} \leq s\right\}
$$

is the set of bases of a discrete bi-polymatroid of rank $q$.

In fact, let $(a, b),(c, d) \in B_{q, s}$ with $a_{i}>c_{i}$ or $b_{k}>d_{k}$. Then for some $j, l$ such that $a_{j}<$ $c_{j}$ or $b_{l}<d_{l}$, we have $(a ; b)-\left(e_{i} ; 0\right)+\left(e_{j} ; 0\right)=\left(a_{1}, \ldots, a_{i}-1, \ldots, a_{j}+1, \ldots, a_{n} ; b_{1}, \ldots, b_{m}\right)$ and $(a ; b)-\left(0 ; e_{k}^{\prime}\right)+\left(0 ; e_{l}^{\prime}\right)=\left(a_{1}, \ldots, a_{n} ; b_{1}, \ldots, b_{k}-1, \ldots, b_{l}+1, \ldots, b_{m}\right)$. Hence, $(a ; b)-$ $\left(e_{i} ; 0\right)+\left(e_{j} ; 0\right) \in B_{q, s}$ and $(a ; b)-\left(0 ; e_{k}^{\prime}\right)+\left(0 ; e_{l}^{\prime}\right) \in B_{q, s}$ by definition. This discrete bipolymatroid is called the discrete bi-polymatroid of Veronese bi-type; we denote it by $P_{q, s}$.

Example 1.7. Let $n=m=2$ and $s=2$. The set of bases of a discrete bi-polymatroid of rank 3 is

$$
\begin{aligned}
B_{3,2}\{(2,0 ; 1,0),(2,0 ; 0,1),(1,1 ; 1,0),(1,1 ; 0,1),(0,2 ; 1,0), \\
(0,2 ; 0,1),(1,0 ; 2,0),(1,0 ; 0,2),(1,0 ; 1,1),(0,1 ; 2,0),(0,1 ; 0,2),(0,1 ; 1,1)\} .
\end{aligned}
$$

The bi-polymatroidal ideal corresponding to the set $B_{q, s}$ is a Veronese bi-type ideal:

$$
L_{q, s}=\sum_{k+r=q} I_{k, s} J_{r, s}, \quad k, r \geq 1, \quad s \leq q,
$$

where $I_{k, s}$ is the Veronese type ideal of degree $k$ in the variables $X_{1}, \ldots, X_{n}$ generated by the set $\left\{X_{1}^{a_{1}} \ldots X_{n}^{a_{n}} \mid \sum_{i=1}^{n} a_{i}=k, 0 \leq a_{i} \leq s\right\}$, and $J_{r, s}$ is the Veronese type ideal of degree $r$ in the variables $Y_{1}, \ldots, Y_{m}$ generated by $\left\{Y_{1}^{b_{1}} \ldots Y_{m}^{b_{m}} \mid \sum_{j=1}^{m} b_{j}=r, 0 \leq b_{j} \leq s\right\}$, 


$$
L_{q, s}=\left(\left\{X_{1}^{a_{1}} \ldots X_{n}^{a_{n}} Y_{1}^{b_{1}} \ldots Y_{m}^{b_{m}} \mid \sum_{i=1}^{n} a_{i}+\sum_{j=1}^{m} b_{j}=q, 0 \leq a_{i}, b_{j} \leq s\right\}\right)
$$

(see [5, 6, 7]).

Remark 1.8. By definition, the ideal $L_{q, s}$ is not trivial for

$$
2 \leq q \leq s(n+m)-1, \quad s \leq q .
$$

Example 1.9. Let $R=K\left[X_{1}, X_{2} ; Y_{1}, Y_{2}\right]$.

$$
\begin{aligned}
L_{4,2}= & I_{3,2} J_{1,2}+I_{1,2} J_{3,2}+I_{2,2} J_{2,2}=I_{3,2} J_{1}+I_{1} J_{3,2}+I_{2} J_{2} \\
= & \left(X_{1}^{2} X_{2} Y_{1}, X_{1}^{2} X_{2} Y_{2}, X_{1} X_{2}^{2} Y_{1}, X_{1} X_{2}^{2} Y_{2}, X_{1} Y_{1}^{2} Y_{2}, X_{2} Y_{1}^{2} Y_{2}, X_{1} Y_{1} Y_{2}^{2}, X_{2} Y_{1} Y_{2}^{2},\right. \\
& \left.X_{1}^{2} Y_{1}^{2}, X_{1}^{2} Y_{1} Y_{2}, X_{1}^{2} Y_{2}^{2}, X_{2}^{2} Y_{1}^{2}, X_{2}^{2} Y_{2}^{2}, X_{2}^{2} Y_{1} Y_{2}, X_{1} X_{2} Y_{1}^{2}, X_{1} X_{2} Y_{2}^{2}, X_{1} X_{2} Y_{1} Y_{2}\right) .
\end{aligned}
$$

Remark 1.10. In general, $I_{k, s} \subseteq I_{k}$, where $I_{k}$ is the Veronese ideal of degree $k$ generated by all monomials in the variables $X_{1}, \ldots, X_{n}$ of degree $k$ (see [10]).

We have $I_{k, s}=I_{k}$ for any $k \leq s$. If $s=1, I_{k, 1}$ is the square-free Veronese ideal of degree $k$ generated by all the square-free monomials in the variables $X_{1}, \ldots, X_{n}$ of degree $k$. Similar considerations are valid for $J_{r, s} \subset K\left[Y_{1}, \ldots, Y_{m}\right]$.

\section{$\S 2$. Monomial Localization}

In this section we study some permanence properties of the bi-polymatroidal ideals of $R$.

Theorem 2.1. Let $L \subset R=K\left[X_{1}, \ldots, X_{n} ; Y_{1}, \ldots, Y_{m}\right]$ be a monomial ideal and $u$ a monomial of $R$. The following conditions are equivalent.

1. $L$ is a bi-polymatroidal ideal.

2. $L: u$ is a bi-polymatroidal ideal for all monomials $u$.

3. L: $u$ is generated in a single degree and has linear quotients for all monomials $u$.

4. $L: u$ has a linear resolution for all monomials $u$.

Proof. $1 \Rightarrow 2$. Let $L$ be a bi-polymatroidal ideal. It is suffices to prove that for all variables $X_{i}$ (respectively, $Y_{k}$ ), $L: X_{i}$ (respectively, $L: Y_{k}$ ) is a bi-polymatroidal ideal of $R$. Set $J=L: X_{i}$. Let $u, v \in G(J)$, then $X_{i} u, X_{i} v \in X_{i} J \subseteq L$. If $\operatorname{deg}_{X_{i}}(u)=$ $\operatorname{deg}_{X_{i}}(v)$, then $X_{i} u$ and $X_{i} v$ have the property described in Definition 1.5. Hence, this property is enjoged by $u$ and $v$. If $\operatorname{deg}_{X_{i}}(u)>\operatorname{deg}_{X_{i}}(v)$, then $X_{i}$ divides $u$. For a variable $X_{t}$ with $\operatorname{deg}_{X_{t}}(u)>\operatorname{deg}_{X_{t}}(v)$, we prove that there exists a variable $X_{j}$ with $\operatorname{deg}_{X_{j}}(v)>$ $\operatorname{deg}_{X_{j}}(u)$ and such that $X_{j}\left(u / X_{t}\right) \in G(J)$. Since $\operatorname{deg}_{X_{t}}\left(X_{i} u\right)>\operatorname{deg}_{X_{t}}\left(X_{i} v\right)$ and $L$ is a bi-polymatroidal ideal, it follows that there exists a variable $X_{j}$ with $\operatorname{deg}_{X_{j}}\left(X_{i} u\right)<$ $\operatorname{deg}_{X_{j}}\left(X_{i} v\right)$ such that $X_{j}\left(X_{i} u / X_{t}\right) \in G(L)$. Then $X_{j}\left(u / X_{t}\right) \in J$. The same result holds true for $\operatorname{deg}_{X_{i}}(u)<\operatorname{deg}_{X_{i}}(v)$.

In similar way, if we set $J=L: Y_{k}$, the claim follows with the same argument. We conclude that $L: u$ is bi-polymatroidal.

$2 \Rightarrow 3$. Since any bi-polymatroidal ideal is generated in a single degree by definition and has linear quotients (Theorem 1.6), the same is true for $L: u$.

$3 \Rightarrow 4$. This follows from the general fact that the ideals generated in a single degree with linear quotients have a linear resolution (see [2, Lemma 4.1]).

$4 \Rightarrow 1$. If $L: u$ has a linear resolution, then it is generated in a single degree for all monomials $u$ of $R$. Let $v, w \in G(L)$ with $\operatorname{deg}_{X_{i}}(v)>\operatorname{deg}_{X_{i}}(w)$ or $\operatorname{deg}_{Y_{k}}(v)>\operatorname{deg}_{Y_{k}}(w)$. We prove that there exists a variable $X_{j}$ such that $\operatorname{deg}_{X_{j}}(w)>\operatorname{deg}_{X_{j}}(v)$ and $X_{j}\left(v / X_{i}\right) \in$ $G(L)$, or there exists $Y_{l}$ such that $\operatorname{deg}_{Y_{l}}(w)>\operatorname{deg}_{Y_{l}}(v)$ and $Y_{l}\left(u / Y_{k}\right)$. 
First, suppose that $\operatorname{deg}_{X_{i}}(v)>\operatorname{deg}_{X_{i}}(w)$, then, by the hypothesis, $L: \frac{v}{X_{i}}$ is generated in the same degree. Since $X_{i} \in G\left(L: \frac{v}{X_{i}}\right)$, it follows that $L: \frac{v}{X_{i}}$ is generated in degree 1 . Since $w / G C D\left(w, \frac{v}{X_{i}}\right) \in L: \frac{v}{X_{i}}$, there exists $z \in G(L)$ such that $X_{j}=z / G C D\left(z, \frac{v}{X_{i}}\right)$ for some $j$ and $X_{j}$ divides $w / G C D\left(w, \frac{v}{X_{i}}\right)$. Then $\operatorname{deg}_{X_{j}}(w)>\operatorname{deg}_{X_{j}}\left(\frac{v}{X_{i}}\right)$. Since $\operatorname{deg}_{X_{i}}(v)>$ $\operatorname{deg}_{X_{i}}(w)$, we have $X_{i} \neq X_{j}$. Hence, $\operatorname{deg}_{X_{j}}(w)>\operatorname{deg}_{X_{j}}\left(\frac{v}{X_{i}}\right)=\operatorname{deg}_{X_{j}}(v)$. Our hypothesis implies that $L$ is generated in a single degree. Hence, $\operatorname{deg}(z)=\operatorname{deg}(v)$. Moreover, since $X_{j}=z / G C D\left(z, \frac{v}{X_{i}}\right)$, it follows that $\operatorname{deg}_{X_{t}}(z) \leq \operatorname{deg}_{X_{t}}\left(\frac{v}{X_{i}}\right)=\operatorname{deg}_{X_{t}}(v)$ for all $t \neq i, j$, $\operatorname{deg}_{X_{j}}(z)=\operatorname{deg}_{X_{j}}\left(\frac{v}{X_{i}}\right)+1=\operatorname{deg}_{X_{j}}(v)+1$, and $\operatorname{deg}_{X_{i}}(z) \leq \operatorname{deg}_{X_{i}}\left(\frac{v}{X_{i}}\right)=\operatorname{deg}_{X_{i}}(v)-1$. Consequently, $z=X_{j}\left(v / X_{i}\right)$, whence $X_{j}\left(v / X_{i}\right) \in G(L)$.

Now we suppose that $\operatorname{deg}_{Y_{k}}(v)>\operatorname{deg}_{Y_{k}}(w)$, then, by the hypothesis, $L: \frac{v}{Y_{k}}$ is generated in the same degree. Using the same argument, we can prove that $Y_{l}\left(v / Y_{k}\right) \in G(L)$ for some $l \in[m]$. The claim follows.

Example 2.2. $R=K\left[X_{1}, X_{2} ; Y_{1}, Y_{2}\right]$. Let

$$
\begin{aligned}
L=L_{3,2}=\left(X_{1} Y_{1} Y_{2}, X_{2} Y_{1} Y_{2}, X_{1} Y_{1}^{2},\right. & X_{2} Y_{1}^{2}, X_{1} Y_{2}^{2}, X_{2} Y_{2}^{2}, \\
& \left.X_{1} X_{2} Y_{1}, X_{1} X_{2} Y_{2}, X_{1}^{2} Y_{1}, X_{1}^{2} Y_{2}, X_{2}^{2} Y_{1}, X_{2}^{2} Y_{2}\right)
\end{aligned}
$$

be a bi-polymatroidal ideal of Veronese bi-type.

Set $u=X_{1}$. Then

$$
L: u=\left(Y_{1} Y_{2}, X_{2} Y_{1} Y_{2}, Y_{1}^{2}, X_{2} Y_{1}^{2}, Y_{2}^{2}, X_{2} Y_{2}^{2}, X_{2} Y_{1}, X_{2} Y_{2}, X_{1} Y_{1}, X_{1} Y_{2}, X_{2}^{2} Y_{1}, X_{2}^{2} Y_{2}\right) \text {. }
$$

Hence,

$$
L: u=\left(Y_{1} Y_{2}, Y_{1}^{2}, Y_{2}^{2}, X_{2} Y_{1}, X_{2} Y_{2}, X_{1} Y_{1}, X_{1} Y_{2}\right)
$$

which is again a bi-polymatroidal ideal.

We denote the set of monomial prime ideals of $R$ by $\mathcal{P}(R)$. Let $\wp \in \mathcal{P}(R)$ be a monomial prime ideal. The monomial localization of a monomial ideal $L$ with respect to $\wp$ is the monomial ideal $L(\wp)$ obtained from $L$ by substituting 1 for the variables that do not belong to $\wp$. More precisely, set $\wp=\left(X_{i_{1}}, \ldots, X_{i_{r}}, Y_{j_{1}}, \ldots, Y_{j_{t}}\right)$; we denote by $L(\wp)$ the monomial ideal in the polynomial ring $K\left[X_{i_{1}}, \ldots, X_{i_{r}}, Y_{j_{1}}, \ldots, Y_{j_{t}}\right]$, where $L(\wp)=L_{C}$ with $C=[n+m] \backslash\left\{i_{1}, \ldots, i_{r}, j_{1}, \ldots, j_{t}\right\}$. If $L$ is a square-free monomial ideal, then $L(\wp)=L: X_{C}$, where $X_{C}=\prod_{i \in C} X_{i}$.

Example 2.3. Let $R=K\left[X_{1}, X_{2} ; Y_{1}, Y_{2}\right]$. Let

$$
\begin{aligned}
L=L_{3,2}=\left(X_{1} Y_{1} Y_{2}, X_{2} Y_{1} Y_{2}, X_{1} Y_{1}^{2},\right. & X_{2} Y_{1}^{2}, X_{1} Y_{2}^{2}, X_{2} Y_{2}^{2}, \\
& \left.X_{1} X_{2} Y_{1}, X_{1} X_{2} Y_{2}, X_{1}^{2} Y_{1}, X_{1}^{2} Y_{2}, X_{2}^{2} Y_{1}, X_{2}^{2} Y_{2}\right)
\end{aligned}
$$

be a bi-polymatroidal ideal of Veronese bi-type.

Set $\wp=\left(X_{2}, Y_{1}, Y_{2}\right)$, We compute the monomial localization of $L$ with respect to $\wp$, i.e., $L(\wp)=L_{C}$ with $C=\{1\}$ :

$L(\wp)=\left(Y_{1} Y_{2}, X_{2} Y_{1} Y_{2}, Y_{1}^{2}, X_{2} Y_{1}^{2}, Y_{2}^{2}, X_{2} Y_{2}^{2}, X_{2} Y_{1}, X_{2} Y_{2}, Y_{1}, Y_{2}, X_{2}^{2} Y_{1}, X_{2}^{2} Y_{2}\right)=\left(Y_{1}, Y_{2}\right)$, obtained from $L$ by substituting 1 for the variable $X_{1}$ that does not belong to $\wp$.

Remark 2.4. For square-free bi-polymatroidal ideals, the monomial localization is a bipolymatroidal ideal, by Theorem 2.1 (because $L(\wp)=L: u$, where $u=\prod_{i \in C} X_{i}$ ).

For the non-square-free bi-polymatroidal ideals $L$, we give the following result.

Theorem 2.5. If $L \subset R$ is a bi-polymatroidal ideal, then:

1) for all $\wp \in \mathcal{P}(R)$, the ideal $L(\wp)$ is a bi-polymatroidal ideal;

2) for all $\wp \in \mathcal{P}(R), L(\wp)$ has linear quotients;

3) for all $\wp \in \mathcal{P}(R), L(\wp)$ has a linear resolution. 
Proof. 1. Let $L \subset R=K\left[X_{1}, \ldots, X_{n} ; Y_{1}, \ldots, Y_{m}\right]$ be a bi-polymatroidal ideal generated in degree $q$. For any $i \in[n+m]$, let $L_{\{i\}}$ be the monomial ideal obtained from $L$ by substituting 1 for the variable with index $i$. Let $\underline{X}^{a} \underline{Y}^{b} \in L$, where $\underline{X}^{a} \underline{Y}^{b}=$ $X_{1}^{a_{1}} \ldots X_{n}^{a_{n}} Y_{1}^{b_{1}} \ldots Y_{m}^{b_{m}}$ and $(a ; b)=\left(a_{1}, \ldots, a_{n} ; b_{1}, \ldots, b_{m}\right) \in \mathbb{Z}_{+}^{n+m}$. Then $L=\left(\left\{\underline{X}^{a} \underline{Y}^{b} \mid\right.\right.$ $(a ; b) \in B\})$. Suppose $i \in[n]$; this means that $(L)_{\{i\}}$ is the monomial ideal obtained from $L$ by substituting 1 for the variable $X_{i}$. It follows that $(L)_{\{i\}}=\left(\left\{\underline{X}^{a^{\prime}} \underline{Y}^{b} \mid(a ; b) \in B\right\}\right)$, where for all $(a ; b) \in B$ we put $\underline{X}^{a^{\prime}} \underline{Y}^{b}=\underline{X}^{a} \underline{Y}^{b} / X_{i}^{a_{i}}$. We prove that $(L)_{\{i\}}$ is a bipolymatroidal ideal.

The first step is to prove that $(L)_{\{i\}}$ is generated in a single degree. If $h_{i}=\max \left\{a_{i} \mid\right.$ $(a ; b) \in B\}$, then we prove that $G\left((L)_{\{i\}}\right)=\left\{\underline{X}^{a} \underline{Y}^{b} / X_{i}^{h_{i}} \mid(a ; b) \in B, a_{i}=h_{i}\right\}$. Let $(c ; d) \in B$, then $c_{i}<h_{i}$. Now we show that there exists $(u, v) \in B$ with $u_{i}=h_{i}$ and such that $\underline{X}^{u^{\prime}} \underline{Y}^{v}$ divides $\underline{X}^{c^{\prime}} \underline{Y}^{d}$. We proceed by induction on $h_{i}-c_{i}$.

If $h_{i}-c_{i}=0$, all is clear. Suppose now that $h_{i}-c_{i}>0$, i.e., $c_{i}<h_{i}$. Let $(a, b) \in B$ with $a_{i}=h_{i}$. By Theorem [1.4, there exists an integer $j \in[n]$ with $a_{j}<c_{j}$ such that $(a ; b)-\left(e_{i} ; 0\right)+\left(e_{j} ; 0\right) \in B$, and, by symmetry, $(c, d)-\left(e_{j} ; 0\right)+\left(e_{i} ; 0\right) \in B$. Set $(z, t)=(c, d)-\left(e_{j} ; 0\right)+\left(e_{i} ; 0\right)$. Consequently, $\underline{X}^{z^{\prime}} \underline{Y}^{t}$ divides $\underline{X}^{c^{\prime}} \underline{Y}^{d}$. Since $h_{i}-z_{i}<h_{i}-c_{i}$, the inductive hypothesis shows that there exists $(u, v) \in B$ with $u_{i}=h_{i}$ such that $\underline{X}^{u^{\prime}} \underline{Y}^{v}$ divides $\underline{X}^{z^{\prime}} \underline{Y}^{t}$. It follows that $\underline{X}^{u^{\prime}} \underline{Y}^{v}$ divides $\underline{X}^{c^{\prime}} \underline{Y}^{d}$, yielding the desired conclusion.

The second step is to show that the set $B^{\prime}=\left\{\left(a^{\prime}, b\right) \mid \underline{X}^{a^{\prime}} \underline{Y}^{b} \in G\left((L)_{\{i\}}\right)\right\}$ is the set of bases of a discrete bi-polymatroid $P^{\prime}$ of rank $q-h_{i}$ on the set $[n+m] \backslash\{i\}$.

For all $\left(a^{\prime} ; b\right) \in B^{\prime}$ we have $\left|\left(a^{\prime}, b\right)\right|=q-h_{i}$. Now we verify the bi-exchange property: if $\left(a^{\prime}, b\right),\left(c^{\prime}, d\right) \in B^{\prime}$ with $a_{k}^{\prime}>c_{k}^{\prime}$, then $k \neq i$. By assumption for $(a, b),(c, d) \in B$, if $a_{k}=a_{k}^{\prime}>c_{k}^{\prime}=c_{k}$, then there exists $l \in[n]$ such that $a_{l}<c_{l}$ and $(a ; b)-\left(e_{k} ; 0\right)+\left(e_{l} ; 0\right) \in$ $B$. Set $(x, y)=(a ; b)-\left(e_{k} ; 0\right)+\left(e_{l} ; 0\right)$; since $a_{i}=c_{i}=h_{i}$, we have $l \neq i$ and $x_{i}=a_{i}$. Hence, $\left(x^{\prime}, y\right) \in B^{\prime}$, i.e., $\left(a^{\prime} ; b\right)-\left(e_{k} ; 0\right)+\left(e_{l} ; 0\right) \in B^{\prime}$ as required. Then $B^{\prime}$ is a discrete bi-polymatroid on the set $[n+m] \backslash\{i\}$. It follows that $(L)_{\{i\}}$ is a bi-polymatroidal ideal.

If $i \in[m]$, the claim follows by the same argument. In all cases $L_{\{i\}}$ is a bipolymatroidal ideal. As a consequence, if $\wp=\left(X_{i_{1}}, \ldots, X_{i_{r}}, Y_{j_{1}}, \ldots, Y_{j_{r}}\right)$ is a prime ideal of $R$, then the monomial localization $L(\wp)=L_{C}$ with $C=[n+m] \backslash\left\{i_{1}, \ldots, i_{r}, j_{1}, \ldots, j_{r}\right\}$ is a bi-polymatroidal ideal.

2. This follows by Theorem 1.6.

3. It is a general fact that the ideals generated in the same degree with linear quotients have a linear resolution (see [2, Lemma 4.1]).

Example 2.6. Let $R=K\left[X_{1}, X_{2} ; Y_{1}, Y_{2}\right]$. Consider the bi-polymatroidal ideal of Example 2.3 .

$$
\begin{aligned}
L=\left(X_{1} Y_{1} Y_{2}, X_{2} Y_{1} Y_{2}, X_{1} Y_{1}^{2}, X_{2} Y_{1}^{2},\right. & X_{1} Y_{2}^{2}, X_{2} Y_{2}^{2}, \\
& \left.X_{1} X_{2} Y_{1}, X_{1} X_{2} Y_{2}, X_{1}^{2} Y_{1}, X_{1}^{2} Y_{2}, X_{2}^{2} Y_{1}, X_{2}^{2} Y_{2}\right)
\end{aligned}
$$

and put $\wp=\left(X_{2}, Y_{1}, Y_{2}\right), C=\{1\}$. Then

$$
L(\wp)=L_{C}=\left(Y_{1}, Y_{2}\right)
$$

which is a bi-polymatroidal ideal of $R$.

\section{REFERENCES}

[1] S. Bandari and J. Herzog, Monomial localization and polymatroidal ideals, European J. Combin. 34 (2013), no. 4, 752-763. MR3010115

[2] A. Conca and J. Herzog, Castelnuovo-Mumford regularity of products of ideals, Collect. Math. 54 (2003), no. 2, 137-152. MR1995137 (2004k:13020)

[3] J. Herzog amd T. Hibi, Discrete polymatroids, J. Algebraic Combin. 16 (2002), no. 3, $239-268$. MR.1957102 (2004c:52017) 
[4] J. Herzog, A. Rauf, and M. Vladoiu, The stable set of associated prime ideals of a polymatroidal ideal, J. Algebraic Combin. 37 (2013), no. 2, 289-312. MR3011344

[5] M. La Barbiera, Integral closure and normality of some classes of Veronese-type ideals, Riv. Mat. Univ. Parma (7) 9 (2008), 31-47. MR2492672(2010f:13004)

[6] _ On a class of monomial ideals generated by s-sequences, Math. Rep. (Bucur.) 12(62) (2010), no. 3, 201-216. MR2744777 (2012a:13035)

[7] _ On standard invariants of bi-polymatroidal ideals, Algebra Colloq. 21 (2014), no. 2, 267274. MR 3192346

[8] _ A note on unmixed ideals of Veronese bi-type, Turkish J. Math. 37 (2013), no. 1, 1-7. MR.3006734

[9] B. Sturmfels, Gröebner bases and convex polytopes, Univ. Lecture Ser., vol. 8, Amer. Math. Soc., Providence, RI, 1996. MR1363949 (97b:13034)

[10] R. H. Villarreal, Monomial algebras, Monogr. Textbooks Pure Appl. Math., vol. 238, Marcel Dekker, Inc., New York, 2001. MR1800904 (2002c:13001)

Department of Mathematics and Informatics, University of Messina, Viale Ferdinando Stagno D'Alcontres 31, 98166 Messina, Italy

E-mail address: monicalb@unime.it

Received 5/SEP/2013

Originally published in English 